\title{
Identification of potential biomarkers and therapeutic targets for human IgA nephropathy and hypertensive nephropathy by bioinformatics analysis
}

\author{
YINGCHUN CUI, SHENGMAO LIU, WENPENG CUI, DAN GAO, WENHUA ZHOU and PING LUO
}

\author{
Department of Nephrology, The Second Hospital of Jilin University, Changchun, Jilin 130041, P.R. China
}

Received May 14, 2016; Accepted April 4, 2017

DOI: $10.3892 / \mathrm{mmr} .2017 .6996$

\begin{abstract}
In order to further elucidate the potential correlations and treatments of $\operatorname{IgA}$ nephropathy $(\operatorname{Ig} \mathrm{AN})$ and hypertensive nephropathy (HT), bioinformatics analysis of IgAN and HT was performed. The mRNA expression profiles of human renal biopsy samples from patients with IgAN, patients with HT and pre-transplant healthy living controls (LD) were downloaded from the Gene Expression Omnibus database. Then, the differentially expressed genes (DEGs) were identified and functions of DEGs were analyzed. Finally, the regulatory networks containing DEGs and related-transcription factors (TFs) were constructed using Cytoscape software. When compared with the LD group, 134 and 188 DEGs were obtained in the IgAN and HT groups, respectively. A total of 39 genes were altered in the HT group when compared with the IgAN group. In addition, 66 genes were shared in the IgAN and HT groups when compared with the LD group, 6 of which [early growth response 1 , activating transcription factor 3 , nuclear receptor subfamily 4 group A member 2 (NR4A2), NR4A1, v-maf avian musculoaponeurotic fibrosarcoma oncogene homolog F and Kruppel like factor 6] were identified as TFs. In addition, DEGs including interleukin (IL) 1 receptor antagonist, collagen type $4 \alpha 2$ chain, IL8, FBJ murine osteosarcoma viral oncogene homolog and somatostatin were enriched in a number of inflammation-associated biological processes, and DEGs including structural maintenance of chromosomes protein 3, v-crk avian sarcoma virus CT10 oncogene homolog and myosin 6 were enriched in non-inflammation-associated biological processes. Therefore, the differentially expressed TF genes and the genes associated with inflammation may be effective as potential therapeutic targets for IgAN and HT.
\end{abstract}

Correspondence to: Dr Wenhua Zhou, Department of Nephrology, The Second Hospital of Jilin University, 218 Ziqiang Street, Changchun, Jilin 130041, P.R. China

E-mail: zhouwenhua01@163.com

Key words: immunoglobulin A nephropathy, hypertensive nephropathy, differentially expressed gene, transcription factor

\section{Introduction}

Chronic kidney disease has become a critical public health concern in China (1). IgA nephropathy (IgAN), the most prevalent type of glomerulonephritis in humans, is characterized by the expansion of the glomerular mesangial matrix with mesangial cell proliferation and/or mononuclear cell infiltration $(2,3)$. This disease has a variable course and can lead to renal failure (4). Hypertensive nephropathy (HT) is a medical condition referring to damage to the kidney due to chronic high blood pressure. IgAN and HT affect a large proportion of the population and induce high levels of pain in patients worldwide (5). Thus, improving strategies for early diagnosis and screening of disease biomarkers in IgAN and HT are key issues.

Previously, a number of biomarkers have been identified for the detection kidney damage at earlier stages of progression, such as proteinuria and serum creatinine. Urinary $\operatorname{IgA}$ and IgG concentrations have been revealed to be higher in patients with $\operatorname{Ig} \mathrm{AN}$ than in healthy controls and in patients with other renal diseases (6). Urinary excretion of interleukin 6 (IL6) has been reported to predict long-term renal outcomes in patients with IgAN (7). In addition, serum galactose-deficient IgA1 and glycan-specific autoantibody levels have been identified as potential candidates for diagnostic biomarkers of IgAN (8). Furthermore, the urinary expressions of transforming growth factor- $\beta$, monocyte chemoattractant protein- 1 and collagen IV are associated with HT (9). However, the underlying association between these factors and a variety of chronic kidney diseases remains largely unknown and there is still a lack of effective treatments for IgAN and HT.

In the present study, in order to gain a deeper insight into the potential correlations and treatments of $\operatorname{IgAN}$ and HT, the mRNA expression profiles of three types of human renal biopsy samples from patients with $\mathrm{IgAN}$ and HT, and pre-transplant healthy living controls (LD) were analyzed to obtain a set of differentially expressed genes (DEGs) and transcription factors (TFs). Finally, functional enrichment was performed to analyze the potential functions of DEGs and to identify candidate biomarkers and treatment targets.

\section{Materials and methods}

Microarray data and data preprocessing. mRNA expression profiles (GSE37460) (10) were extracted from the Gene 
Expression Omnibus database (http://www.ncbi.nlm.nih .gov/geo/)(11). The total microarray contained 69 chips of human renal biopsy samples from 27 patients with IgAN, 15 patients with HT and 27 LD controls (total $\mathrm{n}=69$ ). Renal biopsies were obtained following the collection of informed written consent from all patients and approval from the ethics committee of European Renal cDNA Bank-Kröner-Fresenius biopsy bank (ERCB-KFB) and the specialized subcommittee for internal medicine of the cantonal ethics committee of Zurich (10). RNA from the glomeruli and tubulointerstitial compartments was obtained and processed for hybridization on Affymetrix microarrays, as previously described (10). The expression profiles of 22,283 probe sets from 51 samples (27 IgAN, 15 HT and 9 LD samples) were analyzed by GPL14663 Affymetrix Genechip Human Genome HG-U133A (Affymetrix, Inc., Santa Clara, CA, USA) and 54,675 probe sets from $18 \mathrm{LD}$ samples were analyzed by GPL11670 Affymetrix Human Genome U133 Plus 2.0 Array (Affymetrix, Inc.). To ensure the consistency of the data source and platform, the results from these 51 samples were selected for further analysis. The probe-level data in CEL files were converted into the mRNA expression profiles. Raw data from the 51 samples were preprocessed using the Guanine Cytosine Robust Multi-Array Analysis method $(12)$ with the Affy package $(13,14)$ in R. The Affmetrix Microarray Suited 5 calls (MAS5calls) algorithm was used for Affymetrix CEL file analysis. Then, the probe-level data in CEL files were converted into the mRNA expression profiles.

Screening of DEGs. The Linear Models for Microarray Data package (version 3.22.1; available at http://www.bioconductor .org/packages/release/bioc/html/limma.html) (15) was used to normalize the results and identify the differentially expressed mRNAs between any two groups. The DEGs with the cutoff criteria of $\mid \log _{2}$ fold change $(\mathrm{FC}) \mid>2$ and a Q-value $<0.01$ were considered to be significantly different. In addition, the DEGs shared by the IgAN and HT groups were screened. In order to investigate whether the mRNAs were sample-specific, the Pheatmap package (version 0.7.7; http://cran.r-project .org/web/packages/pheatmap/index.html) (16) in R was used to perform hierarchical clustering by comparing the value of each mRNA in 51 samples.

Functional enrichment analysis of DEGs. The online platform Genomatix (available at http://www.genomatix.de/index.html) was used to identify the enriched functions and pathways in DEGs. $\mathrm{P}<0.01$ was set as the cutoff criterion.

Identification of TFs for DEGs and construction of the regulatory network. Genomatix was also used to identify the enriched TFs of DEGs, screen the key nodes or node pairs and draw the signal pathways that regulate the expression of the DEGs. Finally, the regulatory networks that contained DEGs and TFs were visualized using Cytoscape software (version 3.2.0; http://www.cytoscape.org/) (17).

\section{Results}

Identification of DEGs. Almost 13,096 probe sets were selected from the 22,283 probe sets of 51 samples using the MAS5calls method (Fig. 1). When compared with the LD group, 134 genes (108 up- and 26 downregulated) were significantly modified in the IgAN group (termed IgAN vs. LD; Fig. 1A) and 188 genes (149 genes up- and 39 genes downregulated) were significantly modified in the HT group (termed HT vs. LD; Fig. 1B). In addition, 39 genes (34 genes up- and 5 genes downregulated) were significantly altered in the HT group (termed HT vs. IgAN; Fig. 1C) when compared with the IgAN group. In addition, in order to investigate the differences among the 51 samples, a heat map was obtained to compare their expression values. The hierarchical clustering analysis revealed a clearly distinct expression of 134 DEGs between the IgAN and LD groups (Fig. 2A), 188 DEGs between the HT and LD groups (Fig. 2B) and 39 DEGs between the HT and IgAN groups (Fig. 2C).

To identify further similarities and differences between HT and IgAN, the overlapping DEGs among IgAN vs. LD, HT vs. LD and HT vs. IgAN were analyzed. The results revealed that 66 DEGs were shared in IgAN vs. LD and HT vs. LD (including 52 up- and 14 downregulated DEGs), 10 DEGs were shared in IgAN vs. LD and HT vs. IgAN groups, and 16 DEGs were shared in HT vs. LD and HT vs. IgAN groups (Fig. 3).

Functional annotation of DEGs. Genomatix was used to identify the enriched functions and pathways in DEGs. The results demonstrated that the 66 shared DEGs in IgAN vs. LD and HT vs. LD were enriched in 245 Gene Ontology (GO) terms (including vasculature development, circulatory system development and cardiovascular system development) and significantly enriched in 3 pathways, including hemoglobin's chaperone ( $\mathrm{P}=1.80 \mathrm{e}-4$; Fig. 4A), signal dependent regulation of myogenesis by corepressor microtubule interacting and transport $(\mathrm{P}=2.74 \mathrm{e}-3$; Fig. 4B), and nuclear factor of activated $\mathrm{T}$ cells and hypertrophy of the heart $(\mathrm{P}=7.35 \mathrm{e}-3$; Fig. 4C). In addition, 24 of the 66 shared DEGs were revealed to be associated with kidney cancer, including inhibitor of DNA binding 1 dominant negative helix-loop-helix protein, early growth response 1 (EGRI) and integrin $\alpha 8$ (Table I).

The screened 39 DEGs in the HT vs. IgAN group were divided into three classes: Class I, 10 shared genes in HT vs. IgAN and IgAN vs. LD; class II, 16 shared genes in HT vs. IgAN and HT vs. LD; class III, 13 specific genes HT vs. IgAN (Table II). GO enrichment indicated that IL1 receptor antagonist (ILIRN) and collagen type IV $\alpha 2$ (COL4A2) in class I were enriched in inflammation-associated biological processes such as negative regulation of the IL1-mediated signaling pathway and negative regulation of heterotypic cell-cell adhesion. Structural maintenance of chromosomes 3 (SMC3), $\mathrm{v}$-crk avian sarcoma virus CT10 oncogene homolog $(C R K)$, caldesmon $1(C A L D)$, myosin VI (MYO6), type I DNA topoisomerase (TOP1), ST3 $\beta$-galactoside $\alpha$-2,3-sialyltransferase 1 (ST3GAL1), peptidylprolyl isomerase $\mathrm{G}(P P I G)$, kin Of IRRE like (Drosophila; KIRREL), ankyrin repeat domain 12 (ANKRD12) and tetratricopeptide repeat domain 3 (TTC3) in class II were enriched in noninflammation-associated biological processes including genetic transfer, DNA mediated transformation and actin filament-based process. IL8, FBJ murine osteosarcoma viral oncogene homolog $(F O S)$, pro-platelet 
A

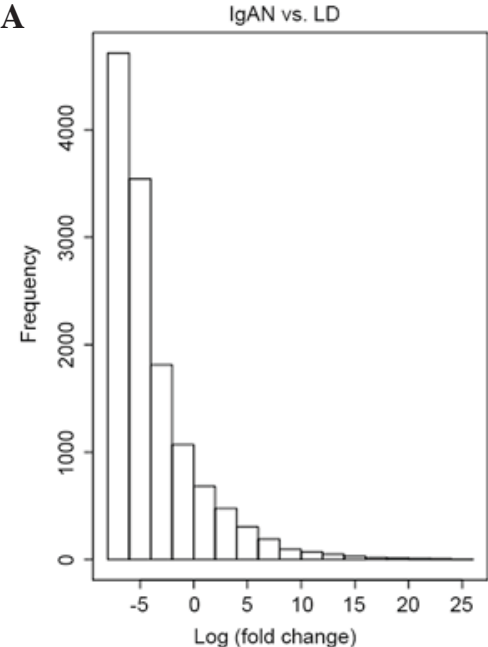

D

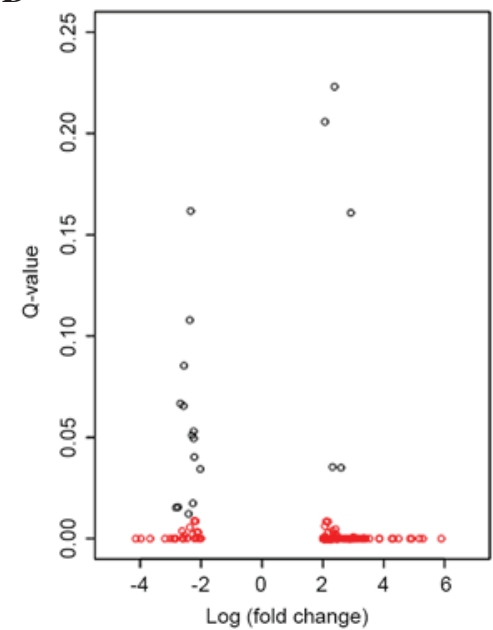

B

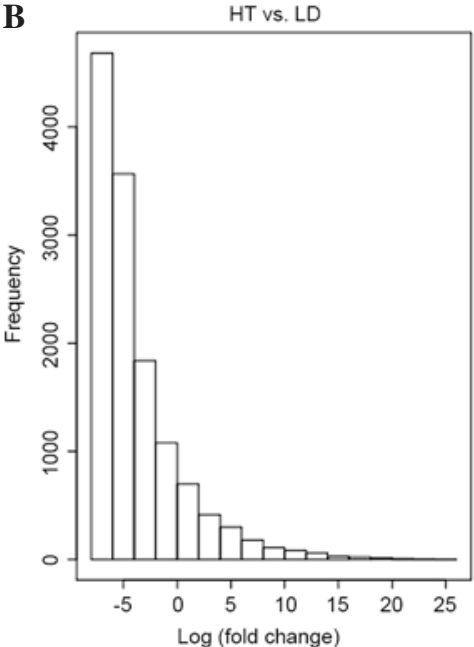

E

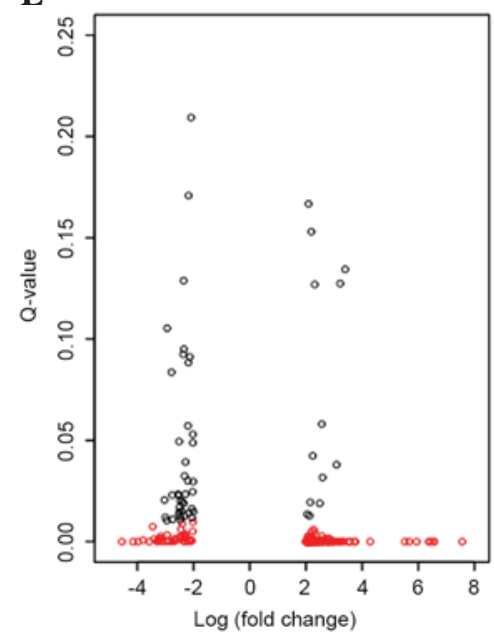

C

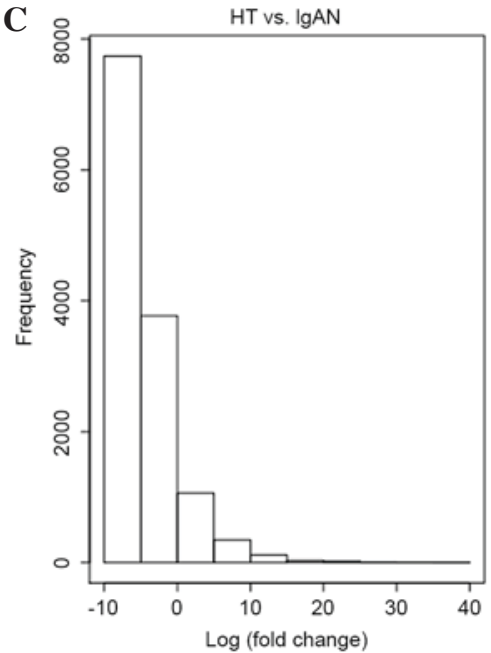

F

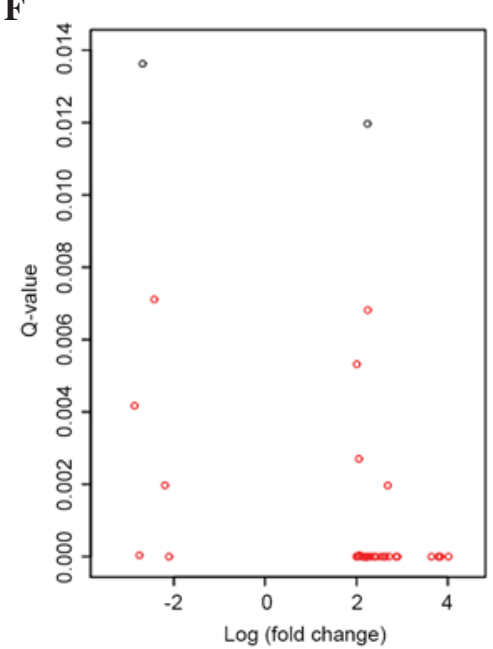

Figure 1. Distribution diagrams of DEG expression levels between any two groups of HT, IgAN and LD. Histograms of the alterations in DEGs expression between (A) IgAN vs. LD, (B) HT vs. LD and (C) HT vs. IgAN. Scatterplots of DEG expression levels between (D) IgAN vs. LD, (E) HT vs. LD and (F) HT vs. IgAN. The red circles represent genes with llogfold changel $>2$ and Q-value $<0.01$. DEG, differentially expressed gene; HT, hypertensive nephropathy; IgAN, IgA nephropathy; LD, healthy controls.

A

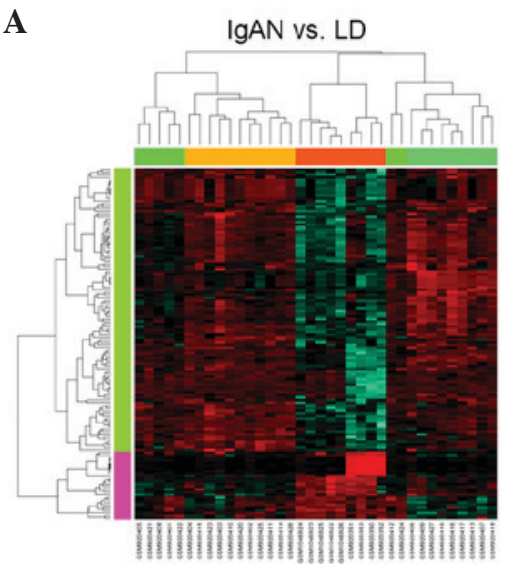

B

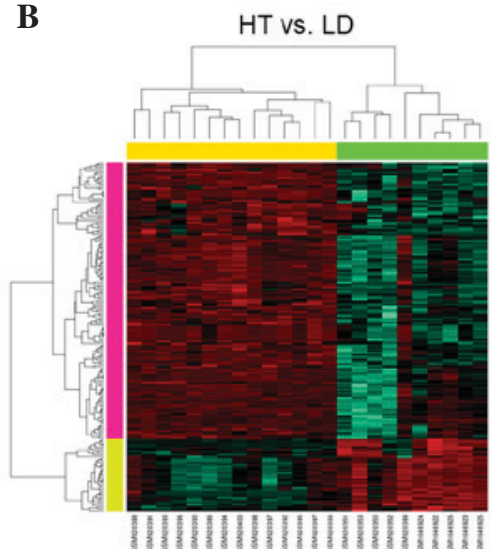

C

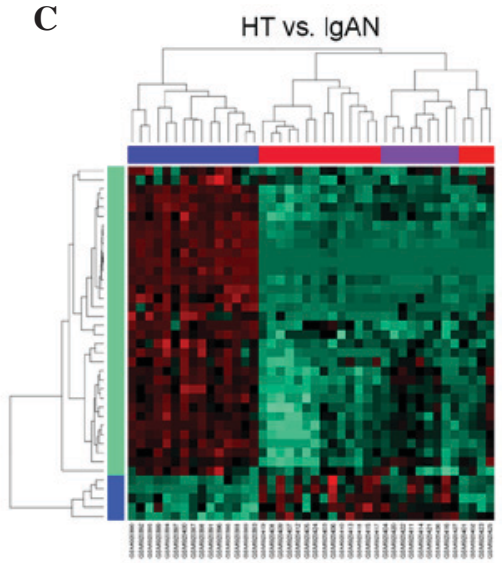

Figure 2. Hierarchical clustering heat maps of each differentially expressed mRNAs. (A) IgAN vs. LD, (B) HT vs. LD and (C) HT vs. IgAN groups. Each column corresponds to a single microarray and each row indicates the expression profile of a single gene. Red and green represent the high and low values in mRNA expression, respectively. IgAN, IgA nephropathy; HT, hypertensive nephropathy; LD, healthy control.

basic protein (chemokine (C-X-C motif) ligand 7 ( $P P B P)$, somatostatin (SST), DIS3 exosome endoribonuclease and 3'-5' exoribonuclease (DIS3), kinesin family member 3B (KIF3B), RNA binding motif protein 25 (RBM25), Rho GTPase 
Table I. Differentially expressed genes associated with different types of cancer.

Associated

cancer tissue

P-value

Genes

Lung $1.03 \times 10^{-3}$

Haematopoietic and lymphoid tissue

Kidneys

\author{
1.98x10 $\quad$ EGR1, FN1, ITGA8, CAMK1, LYN, CTSS, G6PC, SIK1, CASP1, CEACAM1, CYBB \\ ATF3, PXDN, CXCR4, SOSTDC1, FRZB, PPAP2B, RALYL, POLR2E, TIE1 \\ 7.49x10-3 ID1, EGR1, ITGA8, CAMK1, LYN, PYCARD, G6PC, SIK1, CASP1, CYBB, NR4A1, \\ CRIP2, ATF3, RIT1, KLF6, ITGB2, HHEX, CXCR4, RAB31, ACTB, TIE1, NR4A2, \\ $Y W H A H, M A F F$
}

ID1, inhibitor of DNA binding 1 dominant negative helix-loop-helix protein; EGR1, early growth response 1; ITGA8, integrin subunit $\alpha 8$; CAMK1, calcium/calmodulin-dependent protein kinase type 1; LYN, LYN proto-oncogene Src family tyrosine kinase; PYCARD, PYD and CARD domain containing; IGF1, insulin-like growth factor 2; G6PC, glucose-6-phosphatase catalytic subunit; SIK1, salt inducible kinase 1; CASP1, caspase 1; CYBB, cytochrome b-245; CRIP2, cysteine-rich protein 2; ATF3, activating transcription factor 3; RIT1, Ras like without CAAX 1; KLF6, Kruppel-like factor 6; ITGB2, integrin subunit $\beta 2$; HHEX, hematopoietically-expressed homeobox protein; CXCR4, C-X-C chemokine receptor type 4; FRZB, frizzled-related protein; CDH5, cadherin 5; IGFBP5, insulin-like growth factor binding protein 5; RAB31, Ras-reated protein Rab-31; ACTB, actin $\beta$; TIE1, tyrosine kinase with immunoglobulin like and EGF like domains 1; YWHAH, tyrosine 3-monooxygenase/tryptophan 5-monooxygenase activation protein eta; MAFF, v-maf avian musculoaponeurotic fibrosarcoma oncogene homolog F; FN1, fibronectin 1; CTSS, cathepsin S; CEACAM1, carcinoembryonic antigen related cell adhesion molecule 1; PXDN, peroxidasin; SOSTDC1, sclerostin domain containing 1; PPAP2B, phospholipid phosphatase 3; RALYL, RALY RNA binding protein-like; POLR2E, RNA polymerase II subunit E; NR4A1, nuclear receptor subfamily 4 group A member 1.

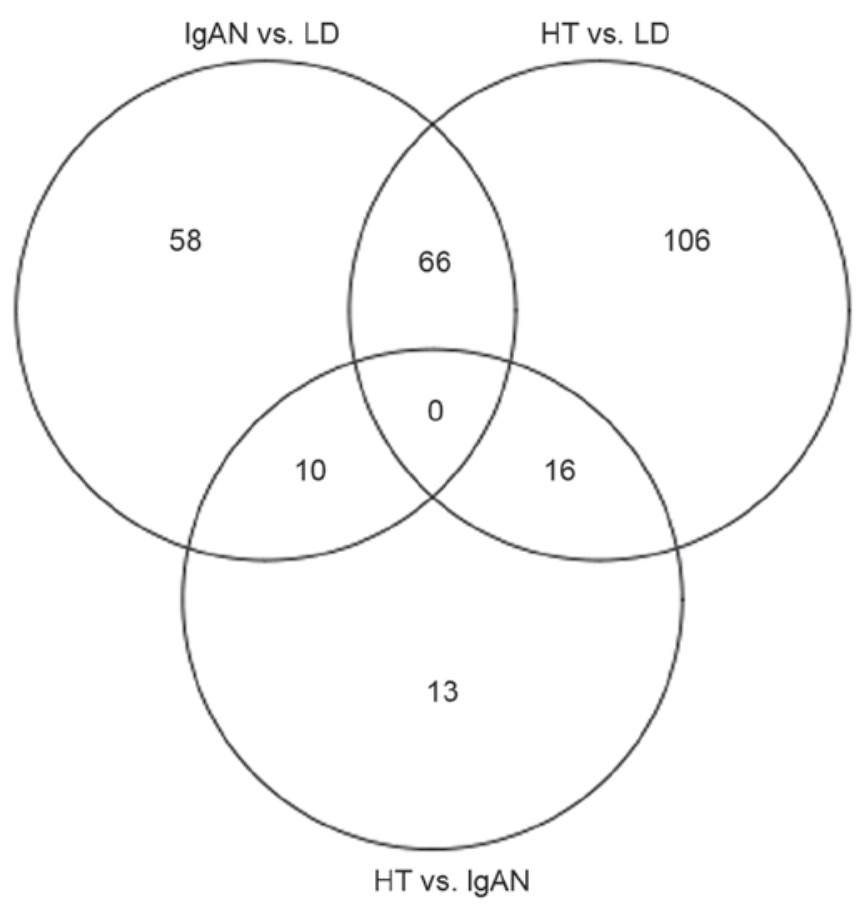

Figure 3. A Venn diagram comparing the differentially expressed genes in the IgAN, HT and LD groups. IgAN, IgA nephropathy; HT, hypertensive nephropathy; LD, healthy control.

activating protein 28 ( $A R H G A P 28)$ and erythrocyte membrane protein band 4.1 like 5 (EPB41L5) in class III were enriched in inflammation-associated biological processes such as the response to bacterium and temperature stimulus, and cryptic unstable transcripts catabolic process.
Identification of TFs and construction of the regulatory network. Genomatix was used to predict the TFs of the 66 shared DEGs in IgAN vs. LD and HT vs. LD, and the 39 genes in HT vs. IgAN. Then, 54 TFs were identified, 6 of which belonged to DEGs including EGR1, activating transcription factor 3 (ATF3), nuclear receptor subfamily 4 group A member 2 (NR4A2), NR4A1, v-maf avian musculoaponeurotic fibrosarcoma oncogene homolog F (MAFF) and Kruppel-like factor 6 (KLF6). In addition, 3 TFs [cAMP responsive element binding protein 5 (CREB5), zinc finger protein 140 (ZNF140) and ZNF85] and 20 TFs (including pirin, TATA-box binding protein associated factor 2 and $\mathrm{NF}$ B repressing factor) were predicted to regulate the genes in class II and class III, respectively. The regulatory networks of DEGs and their TFs are presented in Fig. 5.

\section{Discussion}

Currently, bioinformatics analysis provides a high-efficiency method to study renal disease. In the present study, in order to extend our understanding of IgAN and HT, 39 DEGs in patients with HT were compared with those in patients with IgAN and 66 shared DEGs in IgAN vs. LD and HT vs. LD were identified; their associated TFs were screened as well. These genes may provide a basis for the identification of potential biomarkers. In addition, 6 DEGs were identified as TFs and they may be effective as potential therapeutic targets.

The results demonstrated that 66 genes were significantly altered in patients with IgAN and HT when compared with healthy controls, among which EGR1, ATF3, NR4A2, NR4A1, MAFF and KLF6 were identified as TFs. EGR1, a member of the early growth response family of zinc finger 
A
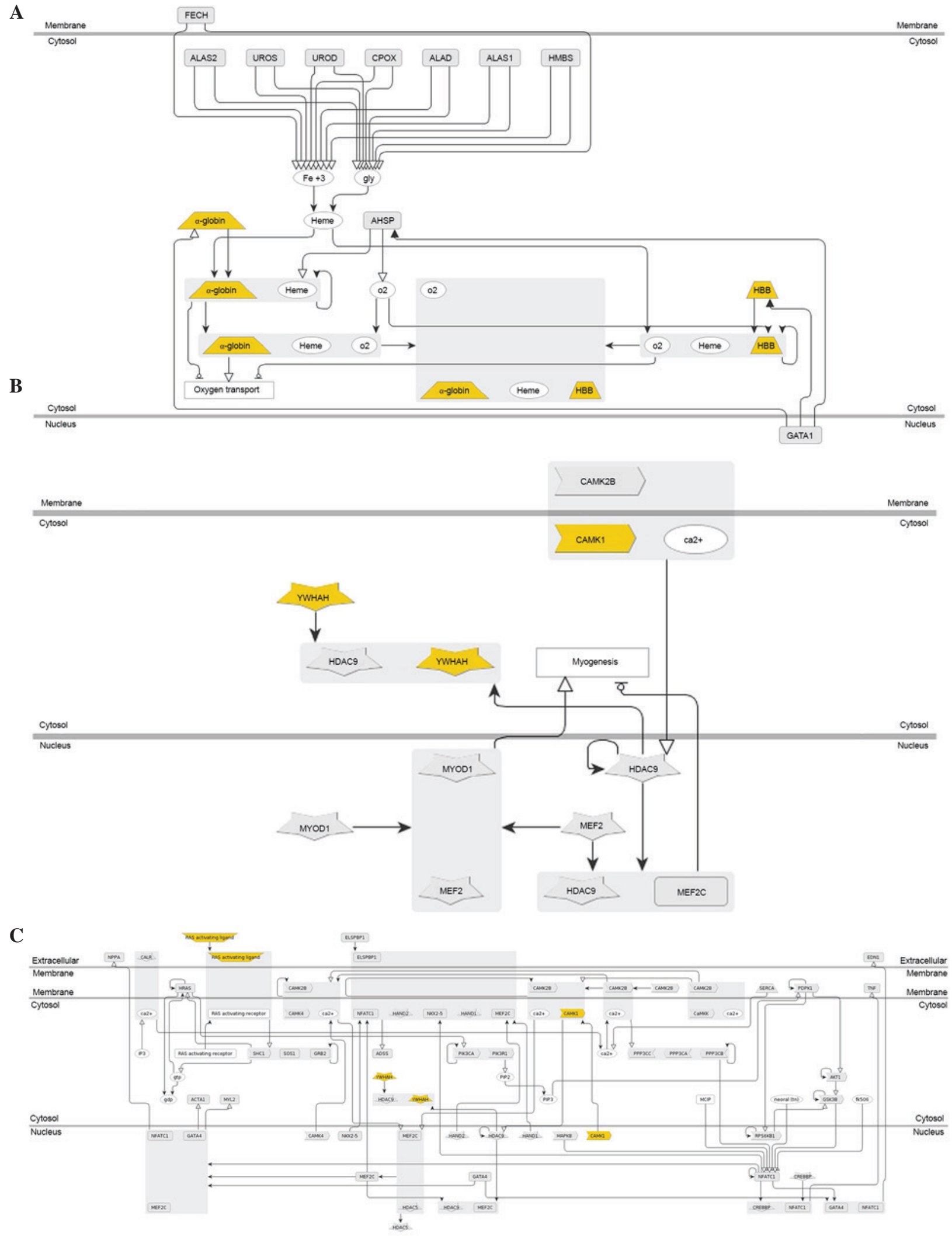

Figure 4. Pathway enrichment analysis. The genes that were significantly enriched in each pathway are marked in yellow. (A) Hemoglobin's chaperone pathway $(\mathrm{P}=1.80 \mathrm{e}-4)$, (B) signal dependent regulation of myogenesis by corepressor microtubule interacting and transport pathway $(\mathrm{P}=2.74 \mathrm{e}-3)$ and $(\mathrm{C})$ nuclear factor of activated $\mathrm{T}$ cells and hypertrophy of the heart pathway ( $\mathrm{P}=7.35 \mathrm{e}-3)$. CAMK1, calcium/calmodulin-dependent protein kinase; YWAH, 14-3-3 eta protein; HBB, haemoglobin subunit $\beta$.

TFs, modulates the regulation of cluster of differentiation 40 ligand (CD40L) expression in megakaryocytes (18). CD40L is important in the development of cardiovascular disease and a number of chronic autoimmune inflammatory diseases that target the vasculature such as chronic kidney disease (19). ATF3, a member of the ATF/CREB family 
Table II. Comparisons between differentially expressed genes in hypertensive nephropathy and immunoglobulin A nephropathy samples.

\section{Class}

Genes

ILIRN, COLAA2

SMC3, CRK, CALD, MYO6, TOP1, ST3GAL1, PPIG, KIRREL, ANKRD12, TTC3

IL8, FOS, PPBP, SST, DIS3, KIF3B, RBM25, ARHGAP28, EPB4IL5
Class I

Class II

Class III

(

IgAN, IgA nephropathy; HT, hypertensive nephropathy; LD, normal control; Class I, 10 shared genes in HT vs. IgAN and IgAN vs. LD; class II, 16 shared genes in HT vs. IgAN and HT vs. LD; class III, 13 specific genes HT vs. IgAN; IL1RN, interleukin 1 receptor antagonist; COL4A2, collagen type IV $\alpha 2$; SMC3, Structural maintenance of chromosomes 3; CRK, v-crk avian sarcoma virus CT10 oncogene homolog; CALD, caldesmon 1; MYO6, myosin VI; TOP1, type I DNA topoisomerase; ST3GAL1, ST3 $\beta$-galactoside $\alpha$-2,3-sialyltransferase 1; PPIG, peptidylprolyl isomerase G; KIRREL, kin Of IRRE like (Drosophila); ANKRD12, ankyrin repeat domain 12; TTC3, tetratricopeptide repeat domain 3; IL8, interleukin 8; FOS, FBJ murine osteosarcoma viral oncogene homolog; PPBP, pro-platelet basic protein chemokine (C-X-C motif) ligand 7; SST, somatostatin; DIS3, DIS3 exosome endoribonuclease and 3'-5' exoribonuclease; KIF3B, kinesin family member 3B; RBM25, RNA binding motif protein 25; ARHGAP28, Rho GTPase activating protein 28; EPB41L5, erythrocyte membrane protein band 4.1 like 5.

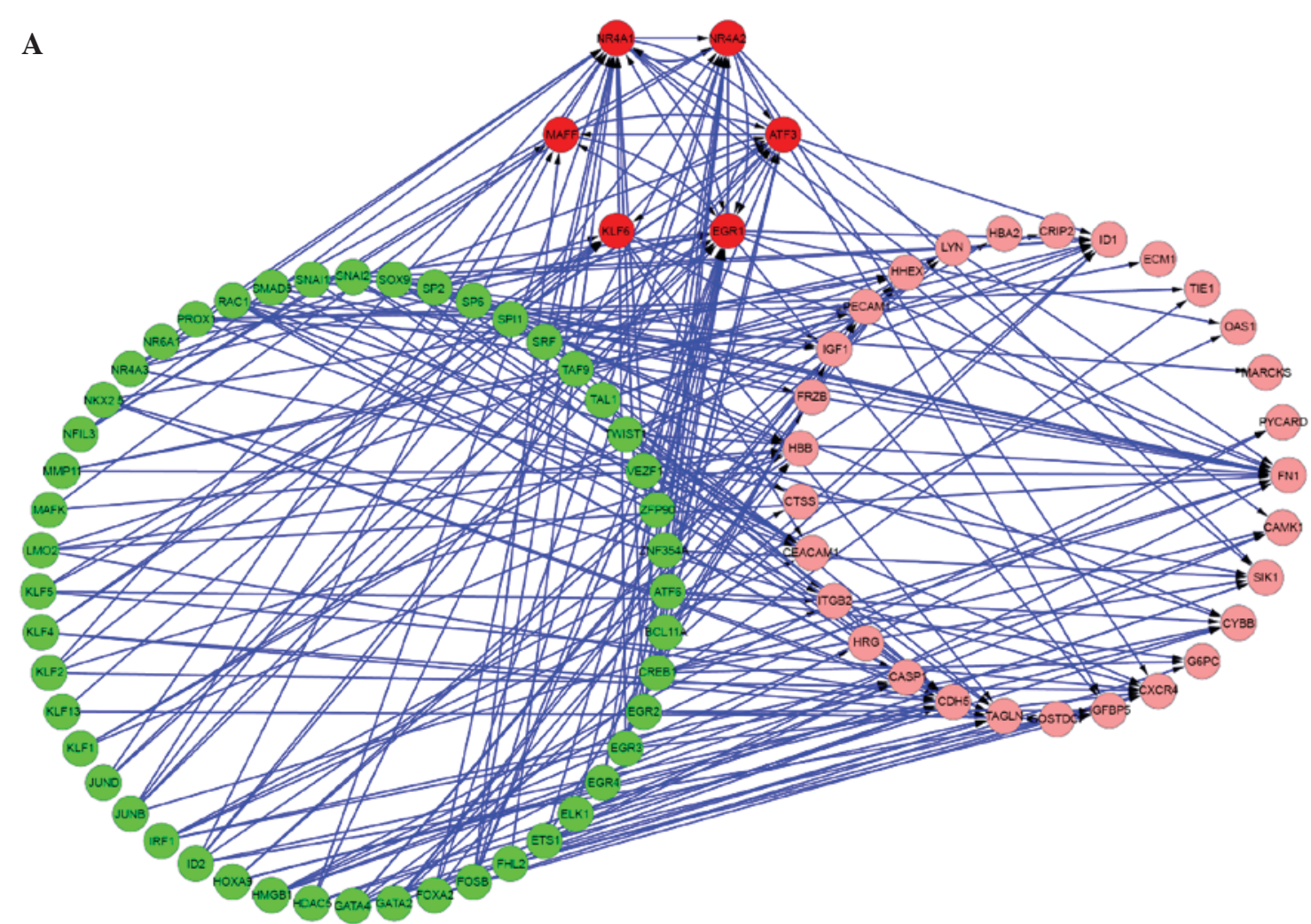

B

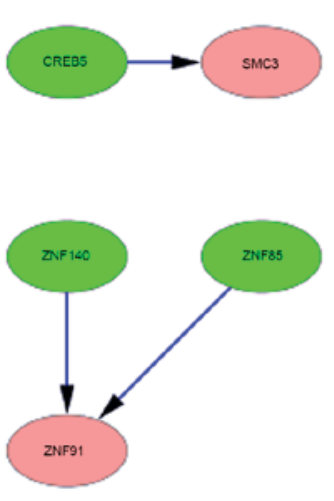

C
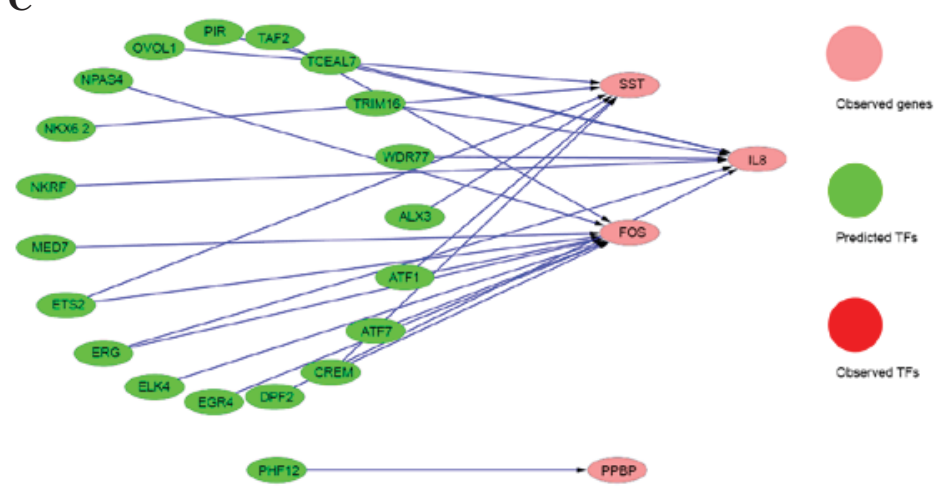

Figure 5. Regulatory networks of DEGs and TFs. Pink nodes indicate the DEGs observed, green nodes indicate the predicted TFs and red nodes indicate the observed TFs that are screened from DEGs. (A) The regulatory network constructed by shared genes in IgAN vs. LD and HT vs. LD groups and their TFs. (B) The regulatory network constructed by specific genes in HT and their TFs. (C) The regulatory network constructed by specific genes in IgAN and their TFs. DEGs, differentially expressed genes; TFs, transcription factors; IgAN, IgA nephropathy; HT, hypertensive nephropathy; LD, normal control. 
of TFs, regulates transcription by binding to DNA sites as a homodimer or heterodimer with c-JUN proteins (20). In addition, EGFR1 and ATF3 have been reported to regulate the expression of inflammation- and immune-associated genes (21). NR4A2 and NR4A1, members of the nuclear hormone receptor subfamily, are involved in cell apoptosis and carcinogenesis (22). MAFF has been associated with the cellular stress response and detected in the kidney (23). KLF6, a DNA-binding protein containing a triple zinc-finger motif, serves a role during kidney development and, more specifically, during the development of the renal collecting duct system (10). Therefore, the results of the present study suggested that EGR1, ATF3, NR4A2, NR4A1, MAFF and KLF6, serve as TFs and serve important roles during the development of human kidney. Thus, they could be used as candidate therapeutic targets for IgAN and HT.

In addition, 39 genes were screened in HT samples and compared with IgAN samples; these genes were then divided into three classes. ILIRN and COL4A2 belong to class I, which were altered in IgAN vs. LD group. IL1RN is involved in autoinflammatory disease (24) and may contribute to the risk of $\operatorname{IgAN}(25,26)$. COL4A2 is a member of the collagen type IV family that may be effective as a marker of renal dysfunction (27). Furthermore, ILIRN and $C O L 4 A 2$ were enriched in a number of inflammation-associated biological processes such as the negative regulation of the IL1-mediated signaling pathway and negative regulation of heterotypic cell-cell adhesion. Genes in class II were also altered in HT vs. LD, including $S M C 3, C R K, C A L D, M Y O 6$, TOP1, ST3GAL1, PPIG, KIRREL, ANKRD12 and TTC3. The results indicated that $S M C 3$ was modulated by CREB5, a TF belonging to the CREB protein family. SMC3 is also involved in sister chromatid cohesion (28). The GO enrichment results demonstrated that genes including SMC3, CRK and MYO6 were enriched in non-inflammation-associated biological processes (including genetic transfer, DNA mediated transformation and actin filament-based process). Thus, the authors speculated that the genes in class II may particulate in the cell cycle of HT cells. In addition, IL8, FOS, PPBP, SST, DIS3, KIF3B, RBM25, ARHGAP28 and $E P B 41 L 5$ in class III were specific DEGs in HT vs. IgAN. IL8, a novel leukocyte chemotactic activating cytokine, has been observed to serve a causative role in acute inflammation by activating neutrophils (29). The expression of SST in IgAN increased and thus may be involved in the pathogenesis of inflammatory renal disease (30). FOS is thought to be involved in the treatment of diabetic nephropathy (31), which is closely associated with HT. Therefore, the results of the present study also suggested that ILIRN and COL $4 A 2$ may be effective biomarkers in patients with $\operatorname{IgAN}$, genes in class II (including $S M C 3, C R K$ and $C A L D$ ) may serve as biomarkers for patients with HT and genes in class III (including $I L 8, F O S$ and $S S T$ ) may be alternative biomarkers for $\operatorname{IgAN}$ and HT.

In conclusion, the present study screened a large number of DEGs and their TFs in IgAN and HT samples, which were then compared with healthy controls by analyzing mRNA expression profiles. The results suggested that EGR1, ATF3, NR4A2, NR4A1, MAFF and KLF6 may be candidate targets for the treatment of $\operatorname{Ig} \mathrm{AN}$ and/or HT. In addition, genes that were screened in HT samples and compared with $\operatorname{IgAN}$ samples, including ILIRN, COL4A2, SMC3, CRK and MYO6, IL8, FOS and $S S T$, may be useful as biomarkers for IgAN and/or HT. However, the majority of candidate biomarkers are still being validated. Therefore, further investigation is required into the role of these biomarkers and targets in appropriate, larger and randomized cohorts, prior to their use in routine clinical screening.

\section{Acknowledgements}

The present study was supported by the Jilin Health and Family Planning Commission Project (grant nos. 3D513J603429 and 2009Z039) and the Foundational Research Programme of Jilin University (grant no. 2012000016).

\section{References}

1. Zhang L, Wang F, Wang L, Wang W, Liu B, Liu J, Chen M, He Q, Liao Y, Yu X, et al: Prevalence of chronic kidney disease in China: A cross-sectional survey. Lancet 379: 815-822, 2012.

2. Tomino Y: Immunopathological predictors of prognosis in $\operatorname{IgA}$ nephropathy. Contrib Nephrol 181: 65-74, 2013

3. Packham D: Prognosis in IgA nephropathy. Nephrology 3: 231-235, 1997.

4. Lee S, Rao VM, Franklin WA, Schiffer MS, Aronson AJ, Spargo BH and Katz AI: IgA nephropathy: Morphologic predictors of progressive renal disease. Hum Pathol 13: 314-322, 1982.

5. Ikee R, Kobayashi S, Saigusa T, Namikoshi T, Yamada M, Hemmi N, Imakiire T, Kikuchi Y, Suzuki S and Miura S: Impact of hypertension and hypertension-related vascular lesions in IgA nephropathy. Hypertens Res 29: 15-22, 2006.

6. Julian BA, Wittke S, Haubitz M, Zürbig P, Schiffer E, Mcguire BM, Wyatt RJ and Novak J: Urinary biomarkers of IgA nephropathy and other IgA-associated renal diseases. World J Urol 25: 467-476, 2007.

7. Harada K, Akai Y,Kurumatani N, Iwano M and Saito Y: Prognostic value of urinary interleukin 6 in patients with $\operatorname{IgA}$ nephropathy: An 8-year follow-up study. Nephron 92: 824-826, 2002.

8. Hastings MC, Moldoveanu Z, Suzuki H, Berthoux F, Julian BA, Sanders JT, Renfrow MB, Novak J and Wyatt RJ: Biomarkers in IgA nephropathy: Relationship to pathogenetic hits. Expert Opin Med Diagn 7: 615-627, 2013.

9. Chaudhary K, Phadke G, Nistala R, Weidmeyer CE, McFarlane SI and Whaley-Connell A: The emerging role of biomarkers in diabetic and hypertensive chronic kidney disease. Curr Diab Rep 10: 37-42, 2010

10. Reich HN, Tritchler D, Cattran DC, Herzenberg AM, Eichinger F, Boucherot A, Henger A, Berthier CC, Nair V, Cohen CD, et al: A molecular signature of proteinuria in glomerulonephritis. PLoS One 5: e13451, 2010

11. Barrett T, Wilhite SE, Ledoux P, Evangelista C, Kim IF, Tomashevsky M, Marshall KA, Phillippy KH, Sherman PM, Holko M, et al: NCBI GEO: Archive for functional genomics data sets-update. Nucleic Acids Res 41 (Database Issue): D991-D995, 2013.

12. Wu Z and Irizarry RA: Preprocessing of oligonucleotide array data. Nat Biotechnol 22: 656-658, 2004.

13. Ihaka R and Gentleman R: R: A language for data analysis and graphics. J Comput Graph Stat 5: 299-314, 1996.

14. Gautier L, Cope L, Bolstad BM and Irizarry RA: Affy-analysis of Affymetrix GeneChip data at the probe level. Bioinformatics 20: 307-315, 2004.

15. Smyth GK: Limma: Linear models for microarray data. In: Bioinformatics and computational biology solutions using $\mathrm{R}$ and Bioconductor. Springer, pp397-420, 2005.

16. Wang L, Cao C, Ma Q, Zeng Q, Wang H, Cheng Z, Zhu G, Qi J, $\mathrm{Ma} \mathrm{H}$, Nian $\mathrm{H}$ and Wang Y: RNA-seq analyses of multiple meristems of soybean: Novel and alternative transcripts, evolutionary and functional implications. BMC Plant Biol 14: 169, 2014. 
17. Kohl M, Wiese S and Warscheid B: Cytoscape: Software for visualization and analysis of biological networks. Methods Mol Biol 696: 291-303, 2011.

18. Crist SA, Elzey BD, Ahmann MT and Ratliff TL: Early Growth response-1 (Egr-1) and nuclear factor of activated T cells (NFAT) cooperate to mediate CD40L expression in megakaryocytes and platelets. J Biol Chem 288: 33985-33996, 2013.

19. Kairaitis L, Wang Y, Zheng L, Tay YC, Wang Y and Harris DC: Blockade of CD40-CD40 ligand protects against renal injury in chronic proteinuric renal disease. Kidney Int 64: 1265-1272, 2003.

20. Tsujino H, Kondo E, Fukuoka T, Dai Y, Tokunaga A, Miki K, Yonenobu K, Ochi T and Noguchi K: Activating transcription factor 3 (ATF3) induction by axotomy in sensory and motoneurons: A novel neuronal marker of nerve injury. Mol Cell Neurosci 15: 170-182, 2000.

21. Pestka JJ: Deoxynivalenol-induced proinflammatory gene expression: Mechanisms and pathological sequelae. Toxins (Basel) 2: 1300-1317, 2010.

22. Li Q, Ke N, Sundaram R and Wong-Staal F: NR4A1, 2, 3-an orphan nuclear hormone receptor family involved in cell apoptosis and carcinogenesis. Histol Histopathol 21: 533-540, 2006.

23. Kimura T, Ogita K, Kumasawa K, Tomimatsu T and Tsutsui T: Molecular analysis of parturition via oxytocin receptor expression. Taiwan J Obstet Gynecol 52: 165-170, 2013.

24. Reddy S, Jia S, Geoffrey R, Lorier R, Suchi M, Broeckel U, Hessner MJ and Verbsky J: An autoinflammatory disease due to homozygous deletion of the IL1RN locus. N Engl J Med 360: 2438-2444, 2009.
25. Syrjänen J, Hurme M,Lehtimäki T, Mustonen J and Pasternack A: Polymorphism of the cytokine genes and IgA nephropathy. Kidney Int 61: 1079-1085, 2002.

26. Liu DJ, Liu Y, Ran LM and Li DT: Genetic variants in interleukin genes and susceptibility to IgA nephropathy: A meta-analysis. DNA Cell Biol 33: 345-354, 2014.

27. W Minz R, Bakshi A, Chhabra S, Joshi K and Sakhuja V: Role of myofibroblasts and collagen type IV in patients of IgA nephropathy as markers of renal dysfunction. Indian J Nephrol 20: 34-39, 2010.

28. Beckouët F, Hu B, Roig MB, Sutani T, Komata M, Uluocak P, Katis VL, Shirahige K and Nasmyth K: An Smc3 acetylation cycle is essential for establishment of sister chromatid cohesion. Mol Cell 39: 689-699, 2010.

29. Harada A, Sekido N, Akahoshi T, Wada T, Mukaida N and Matsushima K: Essential involvement of interleukin-8 (IL-8) in acute inflammation. J Leukoc Biol 56: 559-564, 1994.

30. Bhandari S, Watson N, Long E, Sharpe S, Zhong W, Xu SZ and Atkin SL: Expression of somatostatin and somatostatin receptor subtypes 1-5 in human normal and diseased kidney. J Histochem Cytochem 56: 733-743, 2008.

31. Mao CP and Gu ZL: Puerarin reduces increased c-fos, c-jun, and type IV collagen expression caused by high glucose in glomerular mesangial cells. Acta Pharmacol Sin 26: 982-986, 2005. 